\title{
Dynamic Responses of an Infinite Beam on a Layered Water-Saturated Poroelastic Foundation Under Moving Loads
}

\author{
B. $\mathrm{Xu}^{1,2}$, J.F. $\mathrm{Lu}^{3}$, and J.H. Wang ${ }^{1}$ \\ ${ }^{1}$ Department of Civil Engineer, Shanghai Jiao Tong University, 1954 Hua Shan Road, \\ Shanghai, China \\ 2 Department of Civil Engineering, Nanchang Institute of Technology, 209 Beijing East Road, \\ Nanchang, Jianxi, China \\ 3 Department of Civil Engineering, Jiangsu University, Zhenjiang, Jiangsu, China \\ \{xubin1,wjh417\}@sjtu.edu.cn, ljfdoctor@yahoo.com
}

\begin{abstract}
Based on the Biot's dynamic equation and the dynamic theory of elastic beam, the dynamic responses of an infinite beam resting on a layered water-saturated poroelastic foundation subjected to moving loads is investigated by the transmission and reflection matrices (TRM) method in this study. Applying the triple Fourier transformation, the general solutions for the water-saturated poroelastic foundation and the beam in the transformed domain are derived. Utilizing the continuity conditions between each layer and boundary condition at the surface of the half space, the equivalent stiffness of the layered water-saturated poroelastic foundation is obtained. Time-space domain solutions are obtained by means of IFFT arithmetic. The present methodology was validated by comparing present solutions with some known results. According to numerical results, it can be seen that the occurrence of a softer middle layer enhances the deformation of the beam.
\end{abstract}

Keywords: Moving loads, layered poroelastic foundation, Biot's theory, infinite beam, TRM method.

\section{Introduction}

The dynamic response of an infinite beam resting on a foundation under moving loads has been a hot topic for engineers for a long time, as the model can be conveniently used to simulate the railway subjected to moving train loads and pavements used as roadways. Also, it has been found that most of foundations may consist of one or more layers at some depth under the surface. Thus, a layered half-space is an appropriate approximation for the inhomogeneous soils. The steady-state vibration of a beam supported on an elastic half-space under a moving load has been studied in [1]. The response of an elastic beam on a visco-elastic layer to a moving constant load is investigated in [2]. A review of the literature about solving of a layered half-space is given in [3]. It should be noticed that the transmission and reflection matrix (TRM) method established by Luco and Apsel [4] is a very important method for solving 
dynamic problems of a layered half space. The advantage of the method is that the mismatched exponential terms are eliminated in all the terms of the TRM. However, most research has been mainly treated subsoil as elastic or a layered elastic foundation. The investigation about the layered water-saturated porous medium is in a preliminary stage. It is well known that a saturated soil is two-phase material consisting of soil frame and pore water. Consequently, for a saturated soil, the saturated porous medium model is more realistic than the linear elastic or visco-elastic one. To the best of the authors' knowledge, Jin[5] investigate the dynamic responses of an infinite beam on a poroelastic half space produced by a moving periodic load using a rigorous analytical method.

In this paper, the response of an infinite beam on a layered poroelastic foundation subjected to moving load is studied. Timoshenko beam theory is used to describe the beam, while Biot's theory[6] is adopted to characterize the layered poroelastic soil. Biot's dynamic equations are solved by the Fourier transform method to establish the general solutions of the displacements, the stresses and the pore pressure for the layered soil. Based on the transmission and reflection matrices (TRM) method, the equivalent stiffness of the layered porous foundation is obtained by using the continuity condition between the beam and the layered foundation as well as the continuity conditions at each layer interface. The solutions for the beam and the layered poroelastic foundation are derived in the frequency-wave number domain. By means of the inverse Fourier transform, the time domain solutions of the beam and the poroelastic soil are retrieved from the frequency-wave number solutions. When reduced to some special cases, our solutions agree very well with some known results.

\section{Biot's Equations and Corresponding General Solutions}

The constitutive relations for the porous medium have the form [6]

$$
\left.\begin{array}{l}
\sigma_{i j}=\lambda \delta_{i j} \theta+\mu \varepsilon_{i j}-\alpha \delta_{i j} p \\
p=-\alpha M \theta+M e \\
e=w_{i, i} \\
\theta=u_{i, i}
\end{array}\right\}
$$

where $u_{\mathrm{i}}$ and $w_{\mathrm{i}}(i=1,2,3)$ are the displacement of solid matrix and the infiltration displacement of the pore fluid, respectively; $\sigma_{i j}$ is the stress of the bulk material; $p$ is the pore pressure; $\varepsilon_{i j}$ and $\theta$ is the strain tensor and the dilatation of the solid skeleton; $e$ is the volume of fluid injection into unit volume of bulk material; $\delta_{i j}$ is the Kronecher delta; $\lambda$ and $\mu$ are Lame constants; $\alpha$ and $M$ are Biot's parameters accounting for compressibility of the two-phased material.

The equations of motion for the bulk porous medium and the pore fluid have the form

$$
\left.\begin{array}{l}
\mu u_{i, j j}+\left(\lambda+\alpha^{2} M+\mu\right) u_{j, j i}-\alpha M w_{j, j i}=\rho_{\mathrm{b}} \ddot{u}_{i}+\rho_{\mathrm{f}} \ddot{w}_{i} \\
\alpha M u_{j, j i}+M w_{j, j i}=\rho_{\mathrm{f}} \ddot{u}_{i}+m \ddot{w}_{i}+b \dot{w}_{i}
\end{array}\right\}
$$

where $\rho_{\mathrm{b}}$ and $\rho_{f}$ are mass densities of the bulk material and the pore fluid, $\rho_{\mathrm{b}}=(1-f) \rho_{\mathrm{s}}+f \rho_{\mathrm{s}}$, $\rho_{\mathrm{s}}$ is the density of solid skeleton and $f$ is the porosity of porous medium; $m=\mathrm{a}_{\infty} \rho_{\mathrm{f}} / f$ and $\mathrm{a}_{\infty}$ is tortuosity; $b$ is a parameter account for the viscosity of the pore fluid and the permeability of the porous medium. 
In order to solve Biot's governing equations, two kinds of Fourier transform are involved: the Fourier transform with respect to time and the Fourier transform with respect to horizontal coordinates. In this paper, the Fourier transform for time and the two horizontal coordinates are defined as follows:

$$
\left.\begin{array}{l}
\hat{f}(\omega)=\int_{-\infty}^{\infty} f(t) e^{-i \omega t} d t, \quad f(t)=\frac{1}{2 \pi} \int_{-\infty}^{\infty} \hat{f}(\omega) e^{i \omega t} d \omega \\
\bar{f}(\xi)=\int_{-\infty}^{\infty} f(x) e^{-i \xi x} d x, f(x)=\frac{1}{2 \pi} \int_{-\infty}^{\infty} \bar{f}(\xi) e^{i \xi x} d \xi \\
\tilde{f}(\eta)=\int_{-\infty}^{\infty} f(y) e^{-i \eta y} d y, f(y)=\frac{1}{2 \pi} \int_{-\infty}^{\infty} \tilde{f}(\eta) e^{i \eta y} d \eta
\end{array}\right\}
$$

In this paper, the superimposed symbol ${ }^{\wedge}-\sim$ above a variable denotes the Fourier transform with respect to time $t, x$ - and $y$-directions, respectively.

Performing Fourier transform on Eqs. (1) and (2) and following the procedures as in ref.[5], the general solutions for the homogeneous poroelastic foundation were obtained

$$
\begin{aligned}
& i \xi \hat{\overline{\bar{u}}}_{x}=v_{1}\left(A e^{\gamma_{1} z}+B e^{-\gamma_{1} z}\right)+v_{2}\left(C e^{\gamma_{2} z}+D e^{-\gamma_{2} z}\right)-\gamma_{3}\left(E e^{\gamma_{3} z}-F e^{-\gamma_{3} z}\right)-i \eta\left(G e^{\gamma_{3} z}+H e^{-\gamma_{3} z}\right) \\
& \tilde{\overline{\bar{u}}}_{z}=-\gamma_{1} a_{1}\left(A e^{\gamma_{1} z}-B e^{-\gamma_{1} z}\right)-\gamma_{2} a_{2}\left(C e^{\gamma_{2} z}-D e^{-\gamma_{2} z}\right)+E e^{\gamma_{3} z}+F e^{-\gamma_{3} z} \\
& \hat{\tilde{\bar{u}}}_{y}=-i \eta\left[a_{1}\left(A e^{\gamma_{1} z}+B e^{-\gamma_{1} z}\right)+a_{2}\left(C e^{\gamma_{2} z}+D e^{-\gamma_{2} z}\right)\right]+G e^{\gamma_{3} z}+H e^{-\gamma_{3} z} \\
& \hat{\overline{\bar{w}}}_{z}=\gamma_{1}\left(a_{1} \vartheta+\rho_{f} \omega^{2} / \vartheta\right)\left(A e^{\gamma_{1} z}-B e^{-\gamma_{1} z}\right)+\gamma_{2}\left(a_{2} \vartheta+\rho_{f} \omega^{2} / \vartheta\right)\left(C e^{\gamma_{2} z}-D e^{-\gamma_{2} z}\right)-\vartheta\left(E e^{\gamma_{3} z}+F e^{-\gamma_{3} z}\right) \\
& \hat{\bar{\sigma}}_{z \bar{z}}=\tau_{1}\left(A e^{\gamma_{1} z}+B e^{-\gamma_{1} z}\right)+\tau_{2}\left(C e^{\gamma_{2} z}+D e^{-\gamma_{2} z}\right)+2 \mu \gamma_{3}\left(E e^{\gamma_{3} z}-F e^{-\gamma_{3} z}\right) \\
& i \xi \overline{\bar{\sigma}}_{x z}=-g_{1}\left(A e^{\gamma_{1} z}-B e^{-\gamma_{1} z}\right)-g_{2}\left(C e^{\gamma_{2} z}-D e^{-\gamma_{2} z}\right)-\left(\xi^{2}+\gamma_{3}^{2}\right)\left(E e^{\gamma_{3} z}+F e^{-\gamma_{3} z}\right)-i \gamma_{3} \eta\left(G e^{\gamma_{3} z}-H e^{-\gamma_{3} z}\right) \\
& \hat{\bar{\sigma}}_{y z}=-2 i \eta \gamma_{1} a_{1}\left(A e^{\gamma_{1} z}-B e^{-\gamma_{1} z}\right)-2 i \eta \gamma_{2} a_{2}\left(C e^{\gamma_{2} z}-D e^{-\gamma_{2} z}\right)+i \eta\left(E e^{\gamma_{3} z}+F e^{-\gamma_{3} z}\right)+\gamma_{3}\left(G e^{\gamma_{3} z}-H e^{-\gamma_{3} z}\right) \\
& \hat{\overline{\bar{p}}}=A e^{\gamma_{1} z}+B e^{-\gamma_{1} z}+C e^{\gamma_{2} z}+D e^{-\gamma_{2} z}
\end{aligned}
$$

where $\quad \vartheta=\rho_{f} \omega^{2} /\left(m \omega^{2}-i b \omega\right) \quad, \quad \beta_{1}=\left[\left(m \omega^{2}-\mathrm{i} b \omega\right)\left(\lambda+\alpha^{2} M+2 \mu\right)+\rho \omega^{2} M-2 \alpha M \rho_{f} \omega^{2}\right] /[(\lambda+2 \mu) M] \quad$, $\beta_{2}=\left[\left(m \omega^{2}-\mathrm{i} b \omega\right) \rho \omega^{2} M-\rho_{f}^{2} \omega^{4}\right] /[(\lambda+2 \mu) M] \quad, \quad S^{2}=\left(-\rho_{f} \vartheta+\rho\right) \omega^{2} / \mu \quad, \quad L_{1}^{2}=\frac{1}{2}\left(\beta_{1}-\sqrt{\beta_{1}^{2}-4 \beta_{2}}\right)$, $L_{2}^{2}=\frac{1}{2}\left(\beta_{1}+\sqrt{\beta_{1}^{2}-4 \beta_{2}}\right), \gamma_{j}=\sqrt{\xi^{2}+\eta^{2}-L_{j}^{2}}, \gamma_{3}=\sqrt{\xi^{2}+\eta^{2}-S^{2}}, \tau_{i}=\lambda\left(\chi_{j}-2 \mu a_{j} \gamma_{j}^{2}\right)$, $a_{j}=\left[\lambda\left(\chi_{j}+\mu\right)-\alpha+\vartheta\right] /\left[\left(S^{2}-L_{j}^{2}\right) \mu\right], \chi_{j}=\left[\vartheta M L_{j}^{2}-\rho_{f} \omega^{2}\right] /\left[\rho_{f} \omega^{2}(\alpha-\vartheta) M\right], \quad g_{j}=\chi_{j}+a_{j}\left(\gamma_{j}^{2}-\eta^{2}\right)$, $v_{j}=-\gamma_{j}\left(\chi_{j}+a_{j}\left(\gamma_{j}^{2}+\xi^{2}-\eta^{2}\right)\right)(j=1,2)$.

Note that symbol A, B, C, ....., H are arbitrary functions of $\xi, \eta$ and $\omega$. The radicals $\gamma_{i}(i=1,2,3)$ are selected such that $\operatorname{Re}\left(\gamma_{i}\right) \geq 0$. In addition, $L_{1}$ and $L_{2}$ are the complex wave numbers for the first kind (fast wave) and the second kind (slow wave) dilatational wave, respectively.

\section{The Model and the Equivalent Stiffness for a Beam Resting on a Layered Poroelastic Soil}

The model for an infinite beam resting on $N$ horizontal porous layers overlying a porous half-space subjected to a moving load with a constant velocity $c$ is illustrated in Fig. 1 . The $j$-th porous layer is denoted by the symbol $M_{j}$ and the bottom layer is denoted by the symbol $M_{\mathrm{N}+1}$. The thickness of the $j$-th layer is $h_{j}=z_{j}-z_{j-1}$ and $z_{j}, z_{j-1}$ denote the depth of the upper and lower boundary of the $j$-th layer. 
The following assumptions are made for the beam and the load: (a) The beam is an infinite Timoshenko elastic beam with width $2 a$; (b) The deformation of the beam is small; (b) Both the load and the normal stresses are uniformly distributed over the width of the beam; (c) The contact between the beam and the foundation is smooth.

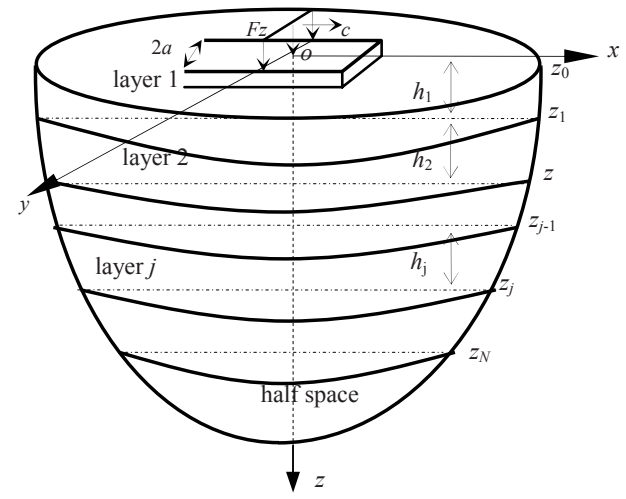

Fig. 1. Model for an infinite beam resting on a layered poroelastic soil

According to Timoshenko beam theory, the equation of motion for the beam is as follows

$$
E I_{z} \frac{\partial^{4} w_{b}(x, t)}{\partial^{4} x}+m_{b} \frac{\partial^{2} w_{b}(x, t)}{\partial^{2} t}=F(x, t)-q(x, t)
$$

where $w_{\mathrm{b}}(x, t)$ is the deflection of the beam, E the Young's modulus of the beam, $\mathrm{I}_{\mathrm{z}}$ the second moment of the area of the beam cross section about its neutral axis, $q(x, t)$ the interaction force between the beam and the surface of the soil, $F(x, t)$ the applied moving load, $x$ the horizontal coordinate along the length of the beam.

The surface load $F(x, t)$ is a line load moving with a constant velocity $c$ and given by the following expression

$$
F(x, t)=F_{z} \delta(x-c t)
$$

where $F_{\mathrm{z}}$ is the magnitude of the force, $\delta(\ldots)$ is the Dirac delta function.

According to the assumptions concerning the beam, the stress, "open pore" boundary conditions for the surface of the layered soil are as follows[7]

$$
\left.\begin{array}{l}
2 a \sigma_{z z}(x, y, 0, t)=q(x, t) H(a-|y|) \\
\sigma_{x z}(x, y, 0, t)=0, \quad \sigma_{y z}(x, y, 0, t)=0, p(x, y, 0, t)=0
\end{array}\right\}
$$

where $\mathrm{H}(\ldots)$ is the unit step function.

Assuming that the centerline of the beam and the surface of the foundation are always in contact, then, the compatibility condition has the form

$$
u_{z}(x, 0,0, t)=w_{b}(x, t)
$$

To analyze the problem, the concept of the "equivalent stiffness" [2] is introduced. According to this concept, to solve the proposed problem, the equivalent stiffness of the 
layered foundation has to be determined in advance. The equivalent stiffness method allows an exact reduction of an original 3-D problem to a 1-D problem by introducing a frequency and wave number dependent complex stiffness $\chi_{e q}^{\text {layer }}$ for the layered foundation. In order to determine the equivalent stiffness of the layered porous soil via the TRM method, for the $j$-th porous layer $M_{j}$, extracting all the positive and negative exponential term $e^{ \pm \gamma_{i} z}(i=1,2,3)$ from general solution for displacement, pore pressure and stress(Eqs.(3)) and combining them with the arbitrary functions $A(\xi, \eta, \omega), \ldots \ldots$, $H(\xi, \quad \eta, \quad \omega)$ which are replaced by $a^{j}(\xi, \eta, \omega) e^{-\gamma_{1}^{j} z_{j}}, \quad b^{j}(\xi, \eta, \omega) e^{\gamma_{1}^{j} z_{j-1}}$, $c^{j}(\xi, \eta, \omega) e^{-\gamma_{2}^{j} z_{j}}, d^{j}(\xi, \eta, \omega) e^{\gamma_{2}^{j} z_{j-1}}, e^{j}(\xi, \eta, \omega) e^{-\gamma_{3}^{j} z_{j}}, f^{j}(\xi, \eta, \omega) e^{\gamma_{3}^{j} z_{j-1}}, g^{j}(\xi, \eta, \omega) e^{-\gamma_{3}^{j} z_{j}}$, $h^{j}(\xi, \eta, \omega) e^{-\gamma_{3}^{j} z_{j-1}}$, the expressions for the displacements, the stresses and the pore pressure of the $j$-th porous layer in the transformation domain are recast as follows

$$
\begin{aligned}
& \boldsymbol{\Psi}^{j}(\xi, \eta, \omega, z)_{8 \times 1}=\left[\begin{array}{ll}
\boldsymbol{D}_{d}^{j}(\xi, \eta, \omega) & \boldsymbol{D}_{u}^{j}(\xi, \eta, \omega) \\
\boldsymbol{S}_{d}^{j}(\xi, \eta, \omega) & \boldsymbol{S}_{u}^{j}(\xi, \eta, \omega)
\end{array}\right]\left[\boldsymbol{W}_{d}^{j}(\xi, \eta, \omega, z)^{T} \boldsymbol{W}_{u}^{j}(\xi, \eta, \omega, z)^{T}\right]^{T}
\end{aligned}
$$

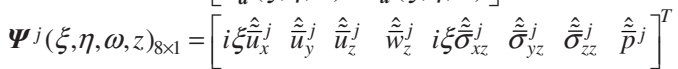

$$
\begin{aligned}
& \boldsymbol{W}_{\boldsymbol{d}}^{j}(\xi, \eta, \omega, z)=\left[b^{j} e^{-\gamma_{1}\left(z-z_{j-1}\right)} d^{j} e^{-\gamma_{2}\left(z-z_{j-1}\right)} f^{j} e^{-\gamma_{3}\left(z-z_{j-1}\right)} h^{j} e^{-\gamma_{3}(z-z j-1)}\right]^{T}
\end{aligned}
$$

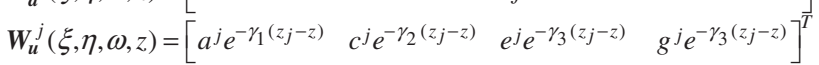

where the superscript $j$ denotes the $j$-th porous layer. The vectors $\boldsymbol{W}_{d}^{j}(\xi, \eta, z, \omega)$, $W_{u}^{j}(\xi, \eta, z, \omega)$ are termed as down-going and up-going wave vector.

In terms of Eq.(9), the down-going and up-going wave vectors are recasted in the following form

$$
\left.\begin{array}{l}
\boldsymbol{W}_{d}{ }^{j}(\xi, \eta, z, \omega)=\boldsymbol{E}^{j}\left(z-z_{j-1}\right) \boldsymbol{W}_{d}{ }^{j}\left(\xi, \eta, z_{j-1}, \omega\right) \\
\boldsymbol{W}_{u}{ }^{j}(\xi, \eta, z, \omega)=\boldsymbol{E}^{j}\left(z_{j}-z\right) \boldsymbol{W}_{u}{ }^{j}\left(\xi, \eta, z_{j}, \omega\right)
\end{array}\right\}
$$

where $\boldsymbol{E}^{j}(\hbar)$ is a $4 \times 4$ identity matrix, $\boldsymbol{E}_{i i}^{j}=e^{-r_{i}^{j} \hbar}(i=1,2,3), \boldsymbol{E}_{44}^{j}=e^{-r_{3}^{j} \hbar}, \boldsymbol{E}_{k q}^{j}=0(k, q=1,2,3,4)$.

According to Deresiewicz and Skalak[7], displacements, the pore pressure, stresses should be continuous at the interfaces. In terms of Eq.(9) and the continuity condition for the $j$-th interface, one has the follows

$$
\begin{gathered}
{\left[\begin{array}{l}
\boldsymbol{W}_{\boldsymbol{d}}{ }^{j+1}\left(\xi, \eta, \omega, z_{j}\right) \\
\boldsymbol{W}_{u}{ }^{j}\left(\xi, \eta, \omega, z_{j}\right)
\end{array}\right]=\left[\begin{array}{ll}
\boldsymbol{T}_{\boldsymbol{d}}{ }^{j} & \boldsymbol{R}_{u}{ }^{j} \\
\boldsymbol{R}_{\boldsymbol{d}}{ }^{j} & \boldsymbol{T}_{u}{ }^{j}
\end{array}\right]\left[\begin{array}{l}
\boldsymbol{W}_{\boldsymbol{d}}{ }^{j}\left(\xi, \eta, \omega, z_{j}\right) \\
\boldsymbol{W}_{u}{ }^{j+1}\left(\xi, \eta, \omega, z_{j}\right)
\end{array}\right]} \\
{\left[\begin{array}{ll}
\boldsymbol{T}_{\boldsymbol{d}}{ }^{j} & \boldsymbol{R}_{u}{ }^{j} \\
\boldsymbol{R}_{\boldsymbol{d}}{ }^{j} & \boldsymbol{T}_{u}{ }^{j}
\end{array}\right]=\left[\begin{array}{ll}
-\boldsymbol{D}_{\boldsymbol{d}}{ }^{j+1} & \boldsymbol{D}_{u}{ }^{j} \\
-\boldsymbol{S}_{\boldsymbol{d}}{ }^{j+1} & \boldsymbol{S}_{\boldsymbol{u}}{ }^{j}
\end{array}\right]^{-1}\left[\begin{array}{ll}
-\boldsymbol{D}_{\boldsymbol{d}}{ }^{j} & \boldsymbol{D}_{u}{ }^{j+1} \\
-\boldsymbol{S}_{\boldsymbol{d}}{ }^{j} & -\boldsymbol{S}_{u}{ }^{j+1}
\end{array}\right]}
\end{gathered}
$$

The $4 \times 4$ matrix $\boldsymbol{R}_{u}{ }^{j}(\xi, \eta, \omega)$ and $\boldsymbol{R}_{d}{ }^{j}(\xi, \eta, \omega)$ in Eq.(11) represent reflection matrices for up-going and down-going $P_{1}, P_{2}, S$ waves incident on the $j$-th interface, while $\boldsymbol{T}_{u}{ }^{j}(\xi, \eta, \omega), \boldsymbol{T}_{d}{ }^{j}(\xi, \eta, \omega)$ denote the transmission matrices for the up-going and down-going $P_{1}, P_{2}, S$ waves incident on the $j$-th interface. For the bottom half space porous medium, one has 


$$
\left.\begin{array}{l}
\boldsymbol{W}_{\boldsymbol{d}}^{N+1}\left(\xi, \eta, \omega, z_{N}\right)=\boldsymbol{T}_{\boldsymbol{d}}^{N} \boldsymbol{W}_{\boldsymbol{d}}^{N}\left(\xi, \eta, \omega, z_{N}\right) \\
\boldsymbol{W}_{\boldsymbol{u}}^{N}\left(\xi, \eta, \omega, z_{N}\right)=\boldsymbol{R}_{\boldsymbol{d}}{ }^{N} \boldsymbol{W}_{\boldsymbol{d}}^{N}\left(\xi, \eta, \omega, z_{N}\right)
\end{array}\right\}
$$

According to the continuity condition on the interface between the $M_{\mathrm{N}}$-th layer and the $M_{N-1}$-th layer, the following relation is obtained

$$
\left.\begin{array}{l}
\boldsymbol{W}_{\boldsymbol{d}}^{N}\left(\xi, \eta, z_{N-1}, \omega\right)=\boldsymbol{T}_{\boldsymbol{d}}^{N-1} \boldsymbol{W}_{\boldsymbol{d}}^{N-1}\left(\xi, \eta, z_{N-1}, \omega\right)+\boldsymbol{R}_{\boldsymbol{u}}^{N-1} \boldsymbol{W}_{\boldsymbol{u}}^{N}\left(\xi, \eta, z_{N-1}, \omega\right) \\
\boldsymbol{W}_{\boldsymbol{u}}^{N-1}\left(\xi, \eta, z_{N-1}, \omega\right)=\boldsymbol{R}_{\boldsymbol{d}}^{N-1} \boldsymbol{W}_{\boldsymbol{d}}^{N-1}\left(\xi, \eta, z_{N-1}, \omega\right)+\boldsymbol{T}_{\boldsymbol{u}}^{N-1} \boldsymbol{W}_{\boldsymbol{u}}^{N}\left(\xi, \eta, z_{N-1}, \omega\right)
\end{array}\right\}
$$

Combination of Eq. (13a) and Eq.(14a) together with the utilization of Eq.(10) yields the generalized transmission matrix for the down-going in the $M_{N}$-th layer incident on the $M_{N-1}$-th interface $(j=N-1)$

$$
\left.\begin{array}{l}
\boldsymbol{W}_{\boldsymbol{d}}^{N}\left(\xi, \eta, z_{N-1}, \omega\right)=\boldsymbol{T}_{\boldsymbol{d}}^{\boldsymbol{g} N-1} \boldsymbol{W}_{\boldsymbol{d}}^{N-1}\left(\xi, \eta, z_{N-1}, \omega\right) \\
\boldsymbol{T}_{\boldsymbol{d}}^{\boldsymbol{g N - 1}}=\left(\boldsymbol{I}-\boldsymbol{R}_{\boldsymbol{u}}^{N-1} \boldsymbol{E}\left(z_{N-1}\right) \boldsymbol{R}_{d}^{N} \boldsymbol{E}\left(z_{N}\right)\right)^{-1} \boldsymbol{T}_{\boldsymbol{d}}^{N-1}
\end{array}\right\}
$$

Note that in Eqs.(14) and (15), the wave vectors $W_{d}^{N}\left(\xi, z_{N-1}, \omega\right), W_{u}^{N}\left(\xi, z_{N-1}, \omega\right)$ at the $M_{N}$-th layer have been expressed in terms of the down-going wave vector $W_{d}^{N-1}\left(\xi, z_{N-1}, \omega\right)$ at the $M_{N-1}$-th layer. Similarly, the up-going and down-going wave vectors at the $j$-th porous layer can also be obtained in terms of the down-going wave vector at the first layer

$$
\left.\begin{array}{l}
\boldsymbol{W}_{d}{ }^{j}\left(\xi, \eta, z_{j-1}, \omega\right)=\boldsymbol{T}_{d e}^{g j-1} \boldsymbol{T}_{d e}^{g j-2} \ldots \boldsymbol{T}_{d e}^{g 2} \boldsymbol{T}_{d}^{g 1} \boldsymbol{W}_{d}^{1}\left(\xi, \eta, z_{1}, \omega\right) \\
\boldsymbol{W}_{u}{ }^{j}\left(\xi, \eta, z_{j}, \omega\right)=\boldsymbol{R}_{d}^{g j} \boldsymbol{W}_{d}{ }^{j}\left(\xi, \eta, z_{j}, \omega\right) \\
\boldsymbol{R}_{d}^{g j}=\boldsymbol{R}_{d}{ }^{j}+\boldsymbol{T}_{u e}{ }^{j} \boldsymbol{R}_{d e}{ }^{j+1} \boldsymbol{T}_{d}^{g j} \\
\boldsymbol{T}_{d e}^{g j}=\left(\boldsymbol{I}-\boldsymbol{R}_{u e}{ }^{j} \boldsymbol{R}_{d e}^{g j+1}\right)^{-1} \boldsymbol{T}_{d e}{ }^{j} \quad j=2,3 \ldots \ldots . . N
\end{array}\right\}
$$

In terms of the surface boundary conditions Eq.(7) and according to Eq. (16), the down-going wave vector for the first layer is obtained

$$
\boldsymbol{W}_{\boldsymbol{d}}{ }^{1}\left(\xi, \eta, z_{0}, \omega\right)=\left[\boldsymbol{S}_{\boldsymbol{d}}^{1}+\boldsymbol{S}_{u}^{1} \boldsymbol{E}^{1}\left(h_{1}\right) \boldsymbol{R}_{d e}^{g 1}\right]^{-1} \hat{\overline{\boldsymbol{Q}}}(\xi, \eta, \omega)
$$

where $\boldsymbol{R}_{d e}^{g 1}$ can be obtained by Eqs.(16) and $\hat{\tilde{\bar{Q}}}(\xi, \eta, \omega)=\left[\begin{array}{llll}0 & 0 & \mathrm{q}(\xi, \omega) \frac{\sin (\eta a)}{\eta a} & 0\end{array}\right]^{T}$.

After the wave vectors in the first layer are determined, it is straightforward to obtain all the variables in the first layer. Also, the vertical displacement of the first layer is obtained by using Eq.(16) and Eq.(17)

$$
\begin{gathered}
\hat{\bar{u}}_{z}\left(\xi, \eta, z_{0}, \omega\right)=\frac{\sin (\eta a)}{\eta a} \phi\left(\xi, \eta, z_{0} \omega\right) \hat{\bar{q}}_{z}(\xi, \omega) \\
\phi\left(\xi, \eta, z_{0}, \omega\right)=-\gamma_{1}^{1} a_{1}^{1}\left(A^{1} e^{-\gamma_{1}^{1} h_{1}}-B^{1}\right)-\gamma_{2}^{1} a_{2}^{1}\left(C^{1} e^{-\gamma_{2}^{1} h_{1}}-D\right)+E^{1} e^{-\gamma_{3}^{1} h_{1}}+F^{1}
\end{gathered}
$$

To implement the compatibility condition (8) and perform a one-dimensional inverse Fourier transform $\eta \rightarrow y$, one has the following equation for the beam in the Fourier transformed domain

$$
\overline{\bar{w}}_{b}(\xi, \omega)\left[-m_{b} \omega^{2}+E I \xi^{4}+\chi_{e q}^{\text {layer }}(\xi, \omega)\right]=-2 \pi F_{z} \delta\left(\omega+\xi_{c}\right)
$$


where $\chi_{e q}^{\text {layer }}(\xi, \omega)=2 \pi / \int_{\infty}^{-\infty} \phi\left(\xi, \eta, z_{0}, \omega\right)[\sin (\eta a) /(\eta a)] d \eta$ is the equivalent stiffness of the layered foundation. As mentioned above, the equivalent stiffness of the layered foundation is a function of the frequency $\omega$ and the wave number $\xi$.

Applying the two-dimensional inverse Fourier transformation on Eq.(20) and using the property of the Dirac's delta function in the integral, the following equation is obtained

$$
w_{b}(x, t)=\frac{F_{z}}{2 \pi} \int_{-\infty}^{\infty} \frac{\delta(\omega+\xi c) e^{i \xi x}}{E I \xi^{4}-m_{b} \omega^{2}+\chi_{e q}^{\text {layer }}(\xi, \omega)} d \xi
$$

It also should be noted that the real $\xi$-axis and $\eta$-axis is free of any singularities for a multi-layered poroelastic medium if the parameter $b$ representing internal friction between the solid and the pore fluid does not vanish. Thus, the infinite integration in Eq. $(20,21)$ with respect to the horizontal wave number $\xi$ and $\eta$ are free of any singularities in the path of integration. In this paper, the FFT method is adopted to perform the inverse Fourier transformation [8]. To computer the inverse transform accurately with the discrete Fourier transform, the integrals must be truncated appropriately to avoid aliasing. We found that an FFT over a grid of 2048 points by 2048 and a range of $-16 \leq \xi, \eta \geq 16$ satisfied both these requirements.

\section{Numerical Results and Discussions}

When the material parameters for each layer are assumed the same values, then, the layered poroelastic half space is reduced to a homogeneous poroelastic half-space. The material parameters for the porous medium are chosen as follows: $\mu=2.0 \times 10^{7} \mathrm{~N} / \mathrm{m}^{2}$, $\lambda=4.0 \times 10^{7} \mathrm{~N} / \mathrm{m}^{2}, \rho_{\mathrm{s}}=2000 \mathrm{~kg} / \mathrm{m}^{3}, f=0.3, \alpha=0.97, M=2.4 \times 10^{8} \mathrm{~N} / \mathrm{m}^{2}, b=1.94 \times 10^{6} \mathrm{~kg} / \mathrm{m}^{3} \mathrm{~s}$, $m=1990 \mathrm{~kg} / \mathrm{m}^{3}$. The parameters for the beam are as follows: $\mathrm{EI}_{\mathrm{z}}=1.28 \times 10^{9} \mathrm{~N} / \mathrm{m}^{2}$, $\mathrm{m}_{\mathrm{b}}=7350 \mathrm{~kg} / \mathrm{m}, \mathrm{a}=2.0 \mathrm{~m}, \mathrm{~F}_{\mathrm{z}}=150 \mathrm{kN}$, respectively. Fig. 2 show the beam displacement curves versus the load velocity for the load at the origin of the coordinate system. In order to validate our method, the solutions of Jin [5] are also shown in Fig.2. From Figure, it shows a very good agreement between our result and that of [5].

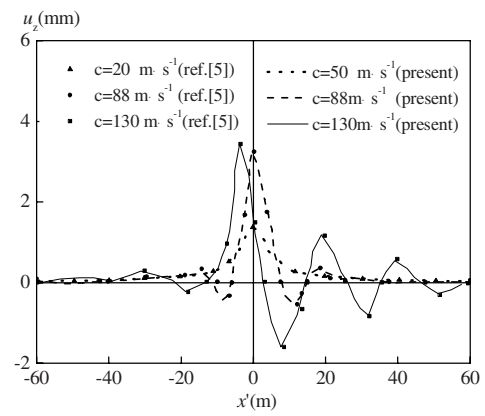

Fig. 2. Comparison of present result with results obtained by ref. [5] 

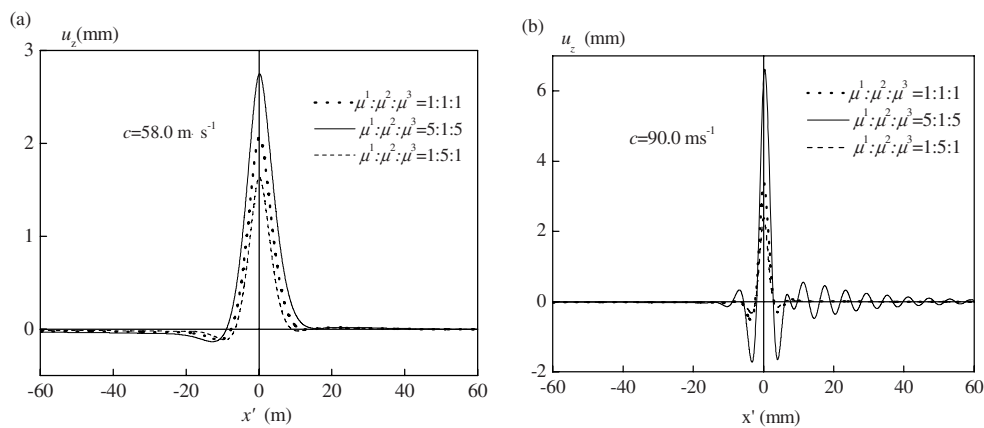

Fig. 3. The deflection of beam subject to moving load on three-layer poroelastic foundation (a) velocity $\mathrm{c}=50 \mathrm{~m} / \mathrm{s} ;$ (b) velocity $\mathrm{c}=90 \mathrm{~m} / \mathrm{s}$

In the second example, the soil is a three layered poroelastic medium: two porous layers and an underlying porous half space. The thicknesses of the two layers are $h^{1}=h^{2}=2.0 \mathrm{~m}$. The following three cases are calculated: A) $\mu^{1}: \mu^{2}: \mu^{3}=1: 5: 1$; B) $\mu^{1}: \mu^{2}$ : $\mu^{3}=5: 1: 5$; C) $\mu^{1}: \mu^{2}: \mu^{3}=1: 1: 1$, where $\mu^{3}=2.0 \times 10^{7} \mathrm{~N} / \mathrm{m}^{2}$. The load moves along the positive $x$-axis. The remaining parameters for each layer and the reference values assume the same values as those in the first example. The load moves with velocities $c=50 \mathrm{~m} / \mathrm{s}$ and $c=90 \mathrm{~m} / \mathrm{s}$ along the positive $x$-direction. The deflections of the beam for the three cases are showed in Figs. 3(a-b), respectively.

It follows from Figs 3(a-b) that the occurrence of a softer middle layer inside the layered foundation leads to a considerable increment of the vertical displacement. Moreover, Fig. $3 \mathrm{~b}$ indicates that the response of the layered medium also exhibits an oscillatory nature.

\section{Conclusions}

The dynamic response of an infinite beam resting on a layered poroelastic foundation subjected to moving loads is addressed in this study. The equivalent stiffness of the layered porous foundation is derived by means of the reflection transmission matrices (TRM) method. Since the TRM solution is free of any mismatched exponential functions, the method can be used in the calculation of the layered porous soil with arbitrary number of layers and arbitrary thickness. The new method was validated by comparing present solutions with some known results. Our calculation indicates that the response of a multi-layered foundation tends to contain higher frequency components and exhibits larger magnitude compared with a homogeneous one.

Acknowledgments. The project is supported by the National Natural Science Foundation of China with grant number No. 50578071. 


\section{References}

1. Madshus C., Kaynia A.M. High-speed railway lines on soft ground: dynamic behaviour at critical train speed. J. Sound Vib.. Vol. 3. (2000) 689-701.

2. Metrikine A.V., Popp K. Steady-state response of an elastic beam on a visco-elastic layer under moving load. Arch. Appl. Mech. Vol. 70. (2000) 399-408.

3. Lu J.F., Hanyga A. Fundamental solution for a layered porous half space subject to a vertical point force or a point fluid source. Comput. Mech. Vol.35(5). (2005) 376-391.

4. Luco J.E., Apsel R.J. On the Green's functions for a layered half-space: Part I. Bull. Seism. Soc. Am. Vol. 73. (1983) 909-929.

5. Jin B. Dynamic displacement of an infinite beam on a poroelastic half space due to a moving oscillating load. Arch. Appl. Mech.. Vol. 74. (2004) 277-287.

6. Biot MA. Mechanics of deformation and acoustic propagation in porous media. J. Appl. Phys.Vol. 33 (1962) 1482-1498.

7. Deresiewicz H., Skalak R. On the uniquness in dynamic poroelasticity. Bull. Seism. Soc. Am. Vol. 53.(1963) 783-788.

8. Oppenheim A.V., Schafer R.W. Discrete-time signal processing. Prentice-Hall, Inc., Englewood Cliffs, NJ.(1999) 